DOI: $10.3901 / J M E .2020 .03 .162$

\title{
无滑动摩擦容积洜的构造*
}

\author{
马 龙 ${ }^{1,2}$ 贺 英 ${ }^{1}$ 周元峰 $^{2}$ 张彩明 2,3
}

(1. 南洋理工大学计算机科学与工程学院 新加坡 639798;

2. 山东大学软件学院 济南 250101;

3. 山东高校未来智能计算协同创新中心 烟台 264025)

\begin{abstract}
摘要: 容积葲是一种通过改变密闭空间的容积来实现流体输送的永。密闭空间容积的改变是通过围成它的机械部件之间的相 对运动实现的。目前的容积洜无法避免这些机械部件之间的滑动摩擦。如果能设计出使用滚动摩擦代替所有滑动摩擦的结构, 则可以让摩擦力大大减小, 从而提高泵的效率和寿命。避免滑动摩擦的同时保证密封却是一个很有挑战性的几何设计问题。 以正八面体为基本结构, 通过在它的棱和面上放置特殊形状的转子和活塞, 成功实现这种构造, 并使用三维几何建模软件模 拟了这种结构的工作过程。该工作不仅从理论上证实了无滑动摩擦的容积原的可实现性, 对高效率容积泵的设计也有着很强 的应用价值。
\end{abstract}

关键词: 容积㫤; 滑动摩擦; 正八面体; 密闭空间

中图分类号: TG156

\section{Construction of Positive Displacement Pump without Sliding Friction}

\author{
MA Long ${ }^{1,2}$ HE Ying $^{1}$ ZHOU Yuanfeng ${ }^{2}$ ZHANG Caiming ${ }^{2,3}$ \\ (1. Computer Science and Engineering, Nanyang Technological University, Singapore 639798; \\ 2. Software College, Shandong University, Jinan 250101; \\ 3. Shandong Co-Innovation Center of Future Intelligent Computing, Yantai 264025)
}

\begin{abstract}
Positive displacement pump is a kind of pump that translate the fluid via changing the volume of closed space. Changing the volume of closed space is based on the relative movements between the mechanical structures composing such space. Now, all the positive displacement pump cannot avoid sliding frictions between these mechanical structures. If the sliding frictions can be replaced by rolling frictions, the forces of frictions can be greatly reduced, so that the efficient and life of pump can be improved. Avoiding frictions while keeping seal is a challenging geometric designing problem. With the regular octahedron as the basic structure, this structure is successfully realized by placing a specially shaped rotor and piston on its ribs and faces, and the working process of this structure is simulated using 3D geometric modeling software. This construction not only show the possibility of realizing a positive displacement pump without slide friction, and has great value for designing a kind of positive displace pump with highly efficient.
\end{abstract}

Key words: positive displacement pump; sliding friction; octahedron; closed space

\section{0 前言}

在人类的生产和生活中, 泵作为一种实现刚体 部件与流体的机械能之间互相转化的装置, 起着不 可替代的作用。原不仅是人类发明的工具，而且在 动物的身体结构中也非常常见, 人和动物的心脏 ${ }^{[1]}$ 就是输送血液的一组复合原。

* 国家自然科学基金青年科学基金(61802228)、国家自然科学基金 (61332015, 61772312)、国家自然科学基金一浙江两化融合联合基金重点 项目(U1609218)、山东省重点研发计划(2017GGX10110)和山东大学基础 研究基金(2018JC030)资助项目。20190406 收到初稿, 20190814 收到修 改稿
泵的工作原理分为两种，有通过改变密闭空间 的容积来实现流体输送的 “容积原” ${ }^{[2]}$, 如心脏和 压缩机; 也有通过在流体内部生成惯性力场来驱动 流体运动的 “压力泵” ${ }^{[3]}$, 如风扇和离心泵 ${ }^{[4]}$ 。

容积洜和压力洜有着完全不同的性质和应用范 围。压力洜结构简单，因而被广泛应用于大流量的 流体输运场合。但压力洜没有用构件隔离流体，因 而没有精确的流量和很大的压差, 这导致它不适合 应用在低速高比例的压缩, 或者有精确流量需求场 合。容积永对通过泵前后的流体有着 “隔离” 作用, 既可以严格控制输出流量, 又能产生较大的压差, 如因而被应用在制冷机 ${ }^{[5]}$ 、计量泵 ${ }^{[6]}$ 等对压力差或流 
量控制有较高要求的领域。由于容积百的工作压力 较大, 确保泵的密封 ${ }^{[7]}$, 防止液体泄漏 ${ }^{[8]}$ 一直是相 关工程领域一个重要的研究方向。

容积洜需要为流体构造可变的密闭空间, 传统 的往复泵 ${ }^{[9]}$, 齿轮原 ${ }^{[10]}$, 螺杆泵 ${ }^{[11]}$, 它们的原理是 利用活塞和气缸, 或者转子和洜壳之间的密闭空间 的变化来实现流体输送。目前所有的容积泵在这些 密闭空间的变化过程中, 构成密闭空间的机械部件 之间的相对滑动 ${ }^{[12]}$ 无法避免, 这种滑动既增加了机 械损耗, 又影响了泵的使用寿命。设计一种利用滚 动摩擦 ${ }^{[13]}$ 代替滑动摩擦的容积原有着非常重要的 理论意义和应用价值。

到目前为止, 所有的容积泵从未真正实现过用 滚动摩擦代替所有的滑动摩擦的设计, 因为这个设 计需要让刚体部件之间在相对滚动的同时确保对流 体密封, 这是一个尚未解决的几何构造难题。

本文先从在二维空间中构造无滑动的可变密闭 空间的构造问题入手, 再把二维空间基于正多边形 的构造拓展到三维空间, 实现了在三维空间中的无 滑动可变密闭空间的构造, 并用几何建模软件 ${ }^{[14]}$ 制 作了三维动画描述它的工作原理。这个构造不仅从 理论方面证明了这个几何构造问题解的存在性, 还 可以用来构造正向容积百实现流体输送和压缩, 可 应用在制冷机, 空气分馏 ${ }^{[15]}$ 等领域, 或者构造反向 容积㫤实现流体的能量利用, 可应用在压缩空气储 能 ${ }^{[16]}$ 、波浪发电 ${ }^{[17]}$ 等领域。

\section{1 相关背景}

常见的容积百有往复百, 螺杆泵和齿轮百, 其 中往复泵是原理最简单的一种容积泵, 它的原理是 利用活塞和气缸构造周期变化的密闭空间, 配合止 回阀的交替开闭实现流体输送(图 1)。

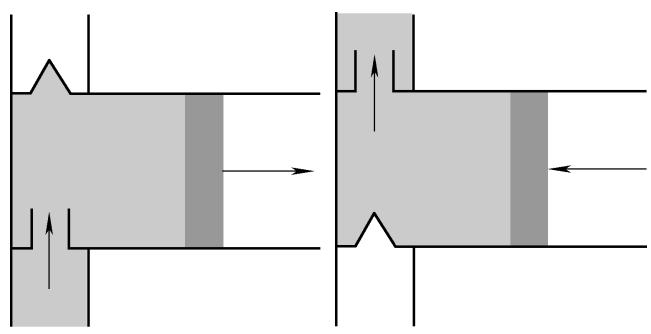

(a) 吸入流体的过程

(b) 送出流体的过程

图 1 往复泵的工作原理

从往复泵的工作过程可以看出, 它的输出流量 $Q$ 和转速 $\omega$ 满足关系

$$
Q \propto \omega
$$

它产生的压力 $p$ 与扭矩 $M$ 满足

$$
p \propto M
$$

往复泵的活塞在往复运动中, 将与气缸产生滑 动摩擦。活塞与气缸之间的正压力不能太小, 否则 会导致流体泄漏, 也不能太大, 否则会使摩擦力过 大造成能量损失。

类似地, 螺杆泵和齿轮洜的转子与泵壳之间也 需要密封, 这种密封同样带来滑动摩擦, 而且密封 效果越好, 滑动摩擦损耗越大。这种矛盾在当前所 有的容积泵的设计中都是存在的。

如果能在容积泵的设计中引入用滚动摩擦代替 滑动摩擦的思想, 则可以彻底解决摩擦力与密封性 能之间的矛盾。由此可见, 设计一种既能避免滑动 摩擦, 同时又能实现对流体密封的结构是一个很有 应用价值的问题。然而确保各部件在相互之间的无 滑动滚动过程中不出现导致流体泄漏的空隙是这项 设计的难点。

\section{2 无滑动可变密闭空间的构造}

为了更方便理解无滑动可变密闭空间的结构, 这里将首先介绍一下如何在二维空间中构造无滑动 可变密闭空间。

\section{1 二维无滑动可变密闭空间}

二维无滑动可变密闭空间的结构如图 2 所示, 它的基本框架是一个正方形, 以正方形的顶点为转 轴放置一个由两个扇形组成的转子, 其中较大的扇 形的半径等于正方形边长的一半。这样在正方形的 上下边上, 组成转子的较大扇形紧密接触; 左右边 上, 组成转子的较小的扇形与长方形活塞紧密接触。 这样，正方形内部形成了密闭空间。

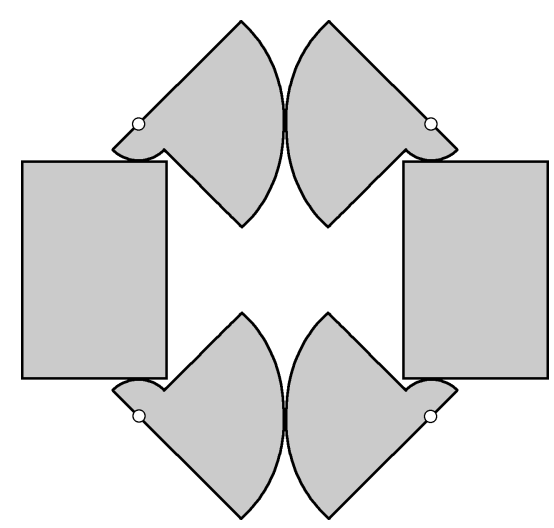

图 2 二维无滑动可变密闭空间

当转子绕轴转动时, 活塞以和它接触的扇形的 线速度相同的速度平动, 因而不会有滑动, 却引起 了正方形内部空间的变化(图 3)。 


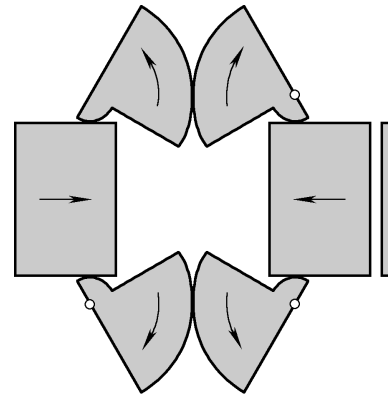

(a) 容积增大的过程

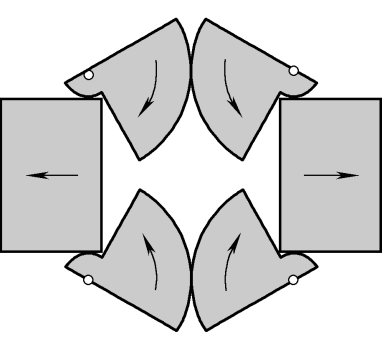

(b) 容积减少的过程
图 3 二维密闭空间无滑动变化过程

由于二维无滑动可变密闭空间的第三个维度不 能密闭, 因而这个构造没有任何实用价值, 但它对 于构造三维无滑动可变密闭空间有着启发作用。如 果这种结构能拓展到三维空间, 则构造无滑动摩擦 的容积泵的问题即可得到解决。

\section{2 基于正八面体的密闭空间}

二维无滑动可变密闭空间的构造利用了正方 形, 在顶点放置了转子。这种构造并不是唯一的, 对于正 $n$ 边形, 只要 $n$ 是偶数, 在顶点处放置转子, 并隔一条边放置一个活塞就可以了。但如果 $n$ 是奇 数, 则不能构造可变密闭空间, 因为当转子转动时, 相邻的顶点上的转子的转动方向必然相反。

对于三维空间, 如果想要以多面体的棱为轴放 置转子, 并保证转子之间能通过紧密接触实现内部 的密闭空间, 则转子的形状必须是由两个共底圆锥 组成的纺雉形。为了能让转子转动中无滑动, 由于 共用顶点的相邻的棱上的转子旋转方向相反, 因此 每个顶点出的发棱数必须为偶数。符合此条件最简 单的多面体即为正八面体 ${ }^{[18]}$, 如图 4a 所示。

在正八面体的每条棱上都以这条棱为旋转轴, 放置由一对轴截面顶角为 $60^{\circ}$ 的共底圆雉组成的 转子(图 $4 \mathrm{~b}$ ), 这些转子共有三组, 每组四个, 围成 一个密闭空间(图 5)所示, 这些转子的转动方向相互 兼容, 使得当转子之间无滑动的相对滚动时, 八面 体有 4 个面内的转子边界线速度由内向外, 另外 4 个面内的转子边界的线速度由外向内。

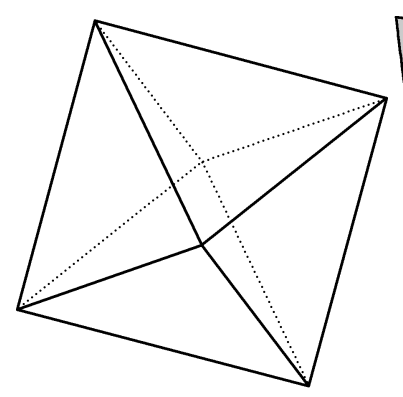

(a) 正八面体

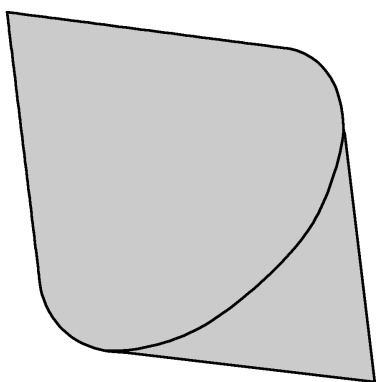

(b) 纺雉形转子
图 4 三维密闭空间构造部件

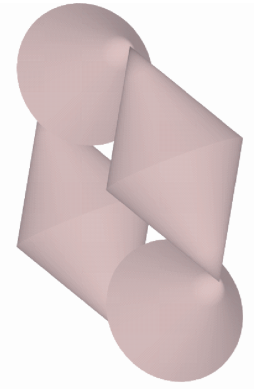

(a) 第一组转子

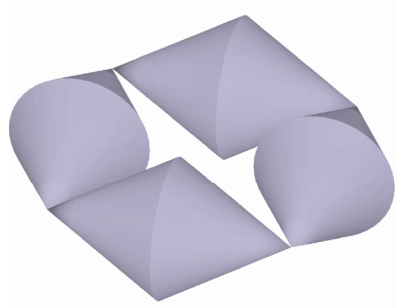

(c) 第三组转子

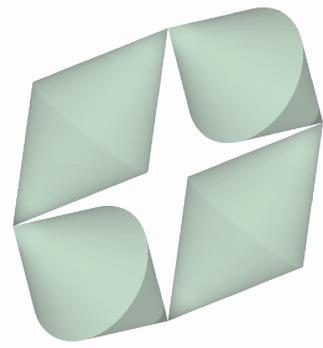

(b) 第二组转子

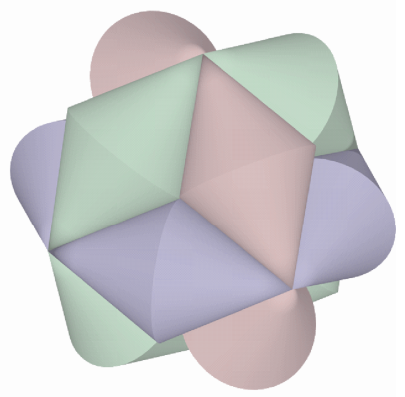

(d) 正八面体密闭空间
图 5 使用纺锥形转子构造的密闭空间

为了计算这个密闭空间的容积, 设转子的底面 半径为 $r$, 由于转子之间必须相切, 八面体的三角 形面的内切圆半径也必须为 $r$ 。设正八面体的棱长 为 $l$, 则根据正三角形的性质, 有关系式

$$
l=2 \sqrt{3} r
$$

把正八面体的中心放在原点, 六个顶点分别放 在坐标轴上(图 6), 设 $t$ 是顶点到原点的距离, 则这 六个顶点的坐标分别为

$$
( \pm t, 0,0),(0, \pm t, 0),(0,0, \pm t)
$$

两个相邻顶点与坐标原点构成等腰直角三角 形，因而有

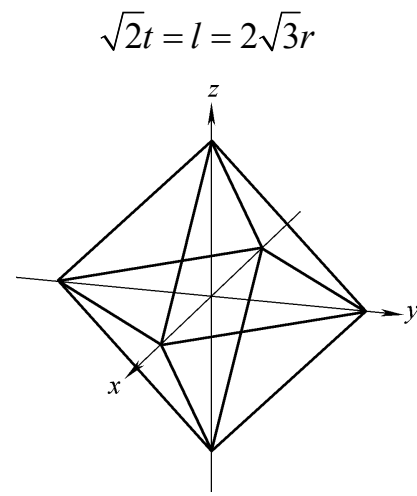

图 6 坐标系中的正八面体

把正八面体视为由上下两个四棱雉组成, 它的 体积为

$$
V_{\text {oct }}=2 \cdot \frac{1}{3} l^{2} t=\frac{2}{3}(2 \sqrt{3} r)^{2} \frac{2 \sqrt{3}}{\sqrt{2}} r=8 \sqrt{6} r^{3}
$$

为了计算转从在这个八面体中占据的空间, 先 
计算一个完整雉体的体积。这个雉体的底面半径为 $r$, 高 $h$ 为八面体棱长 $l$ 的一半

$$
h_{\text {con }}=\frac{l}{2}=\frac{2 \sqrt{3} r}{2}=\sqrt{3} r
$$

雉体的体积为

$$
V_{c o n}=\frac{1}{3} \pi r^{2} h_{c o n}=\frac{1}{3} \pi r^{2} \cdot \sqrt{3} r=\frac{\pi}{\sqrt{3}} r^{3}
$$

圆锥体视为一个直角三角形绕它的直角边旋转 扫过的形状, 则这个直角三角形扫过的体积 $V_{\text {swe }}$ 与它旋转的角度 $\theta$ 成正比, 其中当 $\theta=2 \pi$ 时, $V_{\text {swe } 1}$ 即为 $V_{c o n}$, 因而有

$$
\frac{\mathrm{d} V_{\text {swel }}}{\mathrm{d} \theta}=\frac{V_{c o n}}{2 \pi}=\frac{1}{2 \pi} \frac{\pi}{\sqrt{3}} r^{3}=\frac{1}{2 \sqrt{3}} r^{3}
$$

转子在八面体内部占据的空间对应的角度 $\theta_{\text {max }}$ 即为正八面体的两个相邻面组成的二面角, 取八面 体单位法向量分别为

$$
\left(\frac{1}{\sqrt{3}}, \frac{1}{\sqrt{3}}, \frac{1}{\sqrt{3}}\right)\left(-\frac{1}{\sqrt{3}}, \frac{1}{\sqrt{3}}, \frac{1}{\sqrt{3}}\right)
$$

的两个面, 计算它们组成的二面角得到

$$
\theta_{\max }=\pi-\arccos \frac{1}{3}
$$

由于每个转子包含两个扇雉, 且共有 12 个转 子，因而这个密闭空间的容积为

$$
\begin{gathered}
V_{\text {min }}=V_{\text {oct }}-24 \frac{\mathrm{d} V_{\text {swe }}}{\mathrm{d} \theta} \theta_{\max }= \\
8 \sqrt{6} r^{3}-24 \frac{1}{2 \sqrt{3}} r^{3}\left(\pi-\arccos \frac{1}{3}\right)= \\
\left(8 \sqrt{6}-4 \sqrt{3}\left(\pi-\arccos \frac{1}{3}\right)\right) r^{3} \approx 6.3587 r^{3}
\end{gathered}
$$

后面会看到, 这个容积是本文设计的结构所能 构造出的密闭空间的最小容积。

\section{3 密闭空间的无滑动变化}

使用纺雉形转子虽然可以围成密闭空间, 但转 子的转动并不能引起这个空间的变化。要想让这个 空间成为可变密闭空间, 需要引入三棱柱形活塞。 为了给活塞腾出空间, 转子的形状的设计有所变 化, 这种新的转子是由一对共底的扇雉(图 7a) 和一 个两头尖的扇柱(图 7b)组成(图 7c), 在它的背面会 有空隙(图 7d)。在实际的应用中, 这个空隙会用来 放置固定转子的支架和其它控制转子运动的连动 装置。

为了计算尖头扇柱占据的容积, 把一个完整的 尖头柱形视为由一个圆柱和两个圆雉组成, 圆柱的
底面半径为 $s$ (图 7d), 圆雉的高为

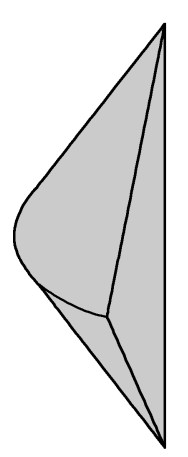

(a) 扇雉

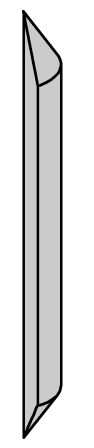

(b) 尖头扇柱

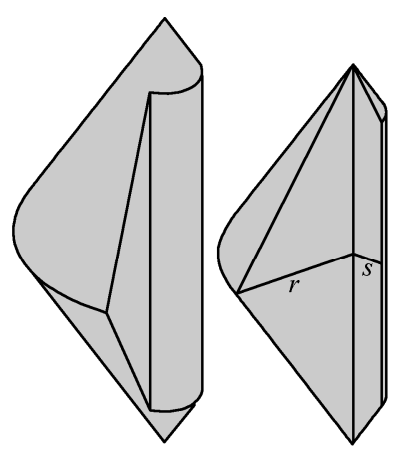

(c) 复合转子 (d) 转子的尺寸
图 7 转子形状组成

$$
h_{t i p}=\sqrt{3} s
$$

圆柱的高为

$$
h_{c y l}=l-2 \sqrt{3} s=2 \sqrt{3}(r-s)
$$

由此得出圆柱的体积

$$
\begin{gathered}
V_{c y l}=\pi s^{2} h_{c y l}= \\
\pi s^{2} \cdot 2 \sqrt{3}(r-s)=2 \sqrt{3} \pi s^{2}(r-s)
\end{gathered}
$$

组成尖头柱形的每个圆雉的体积为

$$
V_{t i p}=\frac{1}{3} \pi s^{2} h_{t i p}=\frac{1}{3} \pi s^{2} \cdot \sqrt{3} s=\frac{\pi}{\sqrt{3}} s^{3}
$$

由此得出尖头柱形的总体积为

$$
V_{a c u-c y l}=V_{c y l}+2 V_{t i p}=\frac{2 \pi}{\sqrt{3}}\left(3 s^{2} r-2 s^{3}\right)
$$

由此来计算当转子的尖头扇柱扫过的体积 $V_{s w 2}$ 关于角度的变化率

$$
\frac{\mathrm{d} V_{s w e 2}}{\mathrm{~d} \theta}=\frac{V_{a c u-c y l}}{2 \pi}=\frac{1}{\sqrt{3}}\left(3 s^{2} r-2 s^{3}\right)
$$

为了保持结构的密闭, 转子的放置要遵守一定 的规则, 要确保转子的空隙朝向外侧, 同时也要保 证在正八面体的八个面中，有四个互不相邻的面完 全由扇雉封住(图 8), 而另外有四个面则会因为扇柱 较细而出现三角形的缺口, 在这此位置装放置三棱 柱形活塞(图 9a)以堵上这些缺口, 从而构造出一个 可变密闭空间(图 9b)。

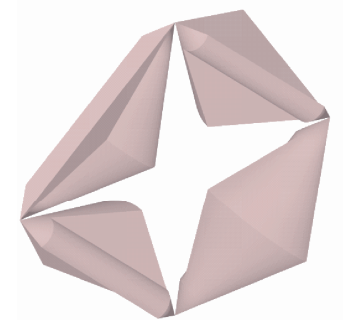

(a) 第一组转子

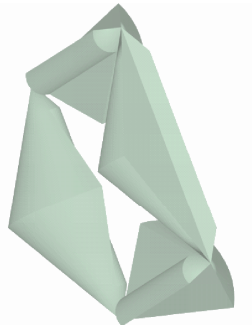

(b) 第二组转子 


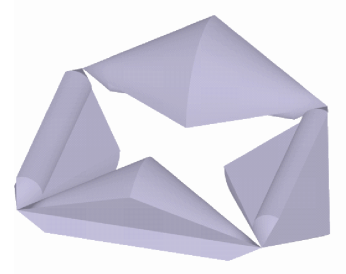

(c) 第三组转子

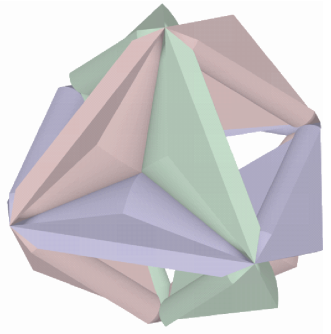

(d) 全部转子

图 8 转子的放置

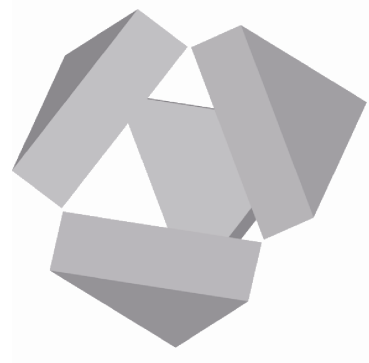

(a) 三棱柱活塞

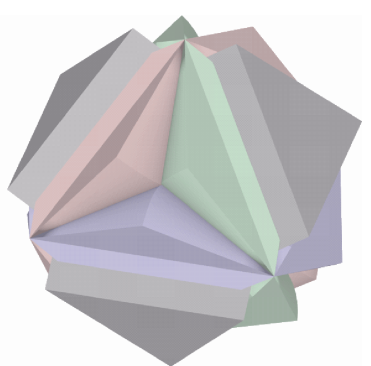

(b) 完整结构
图 9 三棱柱活塞的放置

三棱柱形活塞的移动与转子的转动是同步的, 它们之间是无滑动的滚动, 这即是这种可变密闭空 间的工作过程。当三棱柱形活塞往外移动时, 转子 的扇雉部分会转向八面体内部, 从而占据了更多的 内部空间, 使得密闭空间的容积减小(图 10a); 当三 棱柱形活塞往内移动时, 转子的扇雉部分会转向八 面体外部, 从而占据更少的内部空间, 使得密闭空 间的容积增大(图 10b)。

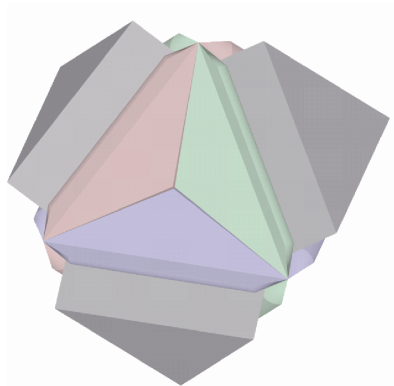

(a) 容积堿少

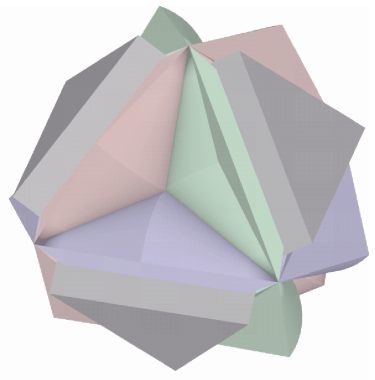

(b) 容积增大
图 10 密闭空间的变化

对于三棱柱形活塞, 它是边长与转子的尖头扇 柱部分的圆柱高相等, 即边长为 $h_{c y l}$ 的等边三角形, 它的面积为

$$
A_{p i s}=\frac{\sqrt{3}}{4} h_{c y l}^{2}=3 \sqrt{3}(r-s)^{2}
$$

由活塞与转子没有相对滑动, 因而每个活塞扫 过的体积随角度的变化律取决于圆柱的半径 $s$ 和活 塞的横截面积 $A_{\text {pis }}$

$$
\frac{\mathrm{d} V_{s w e 3}}{\mathrm{~d} \theta}=A_{p i s} s=3 \sqrt{3}(r-s)^{2} s
$$

根据整体结构由 4 个活塞和 12 个转子组成, 可 以得出容积随转子角度的变化率

$$
\begin{aligned}
& \frac{\mathrm{d} V}{\mathrm{~d} \theta}=24 \frac{\mathrm{d} V_{\text {swe1 }}}{\mathrm{d} \theta}-12 \frac{\mathrm{d} V_{\text {swe } 2}}{\mathrm{~d} \theta}-4 \frac{\mathrm{d} V_{\text {swe } 3}}{\mathrm{~d} \theta}= \\
& 4 \sqrt{3}\left(r^{3}-3 r^{2} s+3 s r^{2}-s^{3}\right)=4 \sqrt{3}(r-s)^{3}
\end{aligned}
$$

由于三角活塞的活动范围有限, 它同时会限制 转子的活动范围。当转子的扇雉部分全部转到八面 体内部时, 三角活塞到了八面体的表面(图 11a), 不 能再继续往外移动, 否则密闭空间将出现开口。三 角活塞也不能无限深入八面体内部, 因为当它们之 间会产生碰撞(图 11b)。

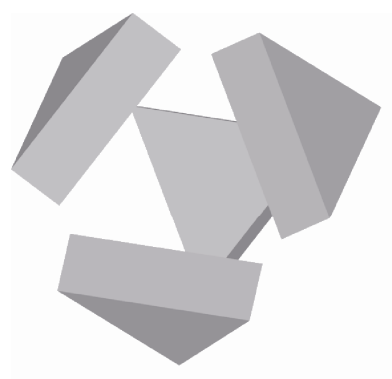

(a) 八面体表面

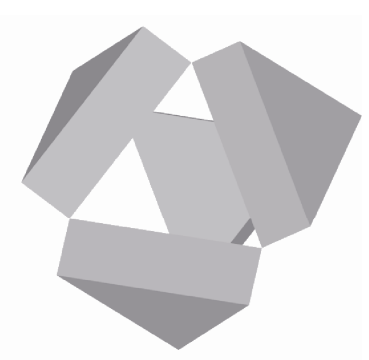

(b) 顶垫碰撞
图 11 三棱柱活塞的活动范围

为了分析活塞的活动范围, 取第一卦限的柱形 活塞，当它在八面体表面时，它的底面方程为

$$
x+y+z=t
$$

取出八面体的这个面, 它是一个正三角形, 活 塞的底面则是与它中心重合的另一个较小的正三角 形(对应尺寸如图 12a)。所选取的八面体面的顶点坐 标分别为

$$
(t, 0,0),(0, t, 0),(0,0, t)
$$

选取活塞底面与 $z$ 轴最接近的顶点, 计算它的 面积坐标 ${ }^{[17]}$, 利用共底三角形的面积性质, 算出它 们对三个顶点的面积坐标(图 12b)如下

$$
\left(\frac{s}{3 r}, \frac{s}{3 r}, \frac{3 r-2 s}{3 r}\right)
$$

因而活塞底面与 $z$ 轴最近的顶点的空间坐标为

$$
\left(\frac{s t}{3 r}, \frac{s t}{3 r}, \frac{(3 r-2 s) t}{3 r}\right)
$$

当活塞沿着该面的法向量移动距离为 $s \theta_{a c t}$ 时, 活塞的顶点发生碰撞, 因而有

$$
\frac{1}{\sqrt{3}} s \theta_{a c t}=\frac{s t}{3 r}
$$

于是导出转子最大旋转角度

$$
\theta_{a c t}=\frac{\sqrt{3} t}{3 r}=\frac{t}{\sqrt{3} r}=\sqrt{2}
$$




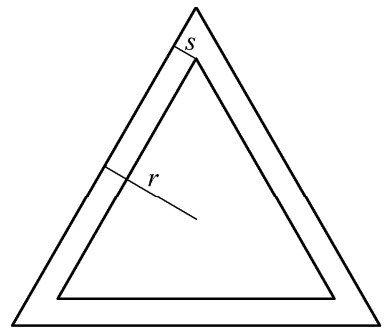

(a) 活塞底面

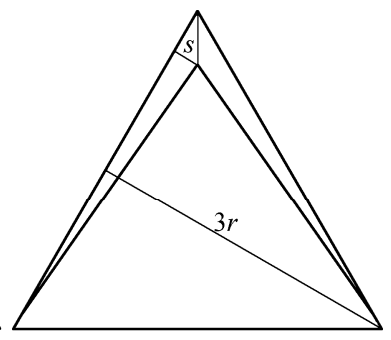

(b) 面积坐标
图 12 活塞位置分析

根据转子的最大旋转角度可以导出密闭空间的 最大容积

$$
\begin{gathered}
V_{\max }=V_{\min }+\frac{\mathrm{d} V}{\mathrm{~d} \theta} \theta_{a c t}= \\
\left(8 \sqrt{6}-4 \sqrt{3}\left(\pi-\arccos \frac{1}{3}\right)\right) r^{3}+ \\
4 \sqrt{6}(r-s)^{3}
\end{gathered}
$$

由此可以看出, 为了增加压缩比, 应该让 $s$ 尽 可能小, 这样三棱柱形活塞会接近全部占据八面体 的面, 它的移动也会很小。不难得到

$$
\lim _{s \rightarrow 0} V_{\max }=\left(12 \sqrt{6}-4 \sqrt{3}\left(\pi-\arccos \frac{1}{3}\right)\right) r^{3} \approx
$$$$
16.1566 r^{3}
$$

把上面的结果和最小体积作比较, 得到极限压 缩比约为 2.54 。要想有更大的压缩比, 可以在内部 填充占据空间的构件。

\section{4 实用密封性质的讨论}

在实际应用的装配过程中, 由于材料的弹性可 能会让部件的变形, 导致尖端产生泄露的风险。为 了避免泄露, 转子的轴部外侧用弹簧支撑, 确保转 子紧密接触, 并在尖端处增加装配应力和保护构件, 这样可以消除变形对密闭性产生的影响。

与气缸活塞结构相比, 这种结构虽然为避免滑 动摩擦付出了部件的形状和数量较为复杂的代价, 但是滚动摩擦力有随着尺寸的增大而减小的性质, 因而该结构的无滑动摩擦的性质适用于大容积大尺 寸的领域。通过对材料滑动摩擦性质和滚动摩擦性 质的比较可以得到, 在转子半径 $1 \mathrm{~cm}$ 左右的数量级 下, 钢质结构的损耗是同样压力下的气缸活塞结构 损耗的 $1 \%$, 在更大的尺寸中, 该结构用滚动摩擦 代替滑动摩擦的优势会更加突出。

\section{3 几何建模与 $3 \mathrm{D}$ 模拟}

为了更好地说明这种无滑动摩擦密闭空间结构 的工作原理, 这里使用三维建模软件 Blender 对转
子的形状构造和位置摆放做相关的说明, 并加入部 分动画截图。

在 Blender 中首先构造转子两端的圆雉, 把圆 雉的底面删去, 只保留圆雉侧面的 2/3(图 13a), 然 后依次加入扇柱面(图 13b), 两个雉面(图 13c、13d), 最后用多边形把开放的面彻底封住, 构成一个实体 (图 13e), 从而实现对转子的建模。

在整个结构中, 转子的中心要放在八面体的棱 的中点处, 并设置好三个欧拉角(图 14)。其中转子 绕自己轴旋转的自转角是在动画中唯一变化的量。

从动画(图 15)中可以看出, 在运动中, 转子之 间也保持着完美的对称性, 活塞也在同步运动。因 此它们之间的接触点的确没有滑动摩擦, 而且始终 保持内部空间的密闭, 从而实现容积原的功能。

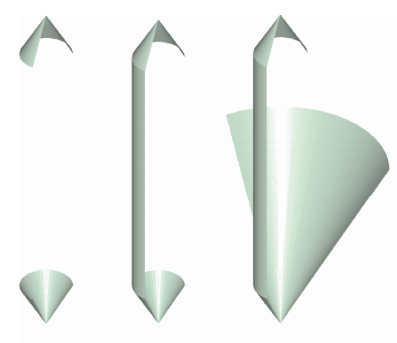

(a) (b)

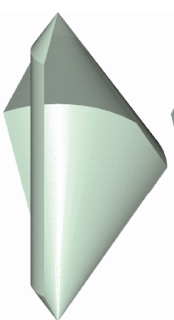

(d)

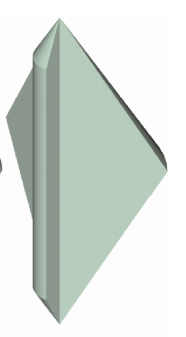

(e)
图 13 转子的建模
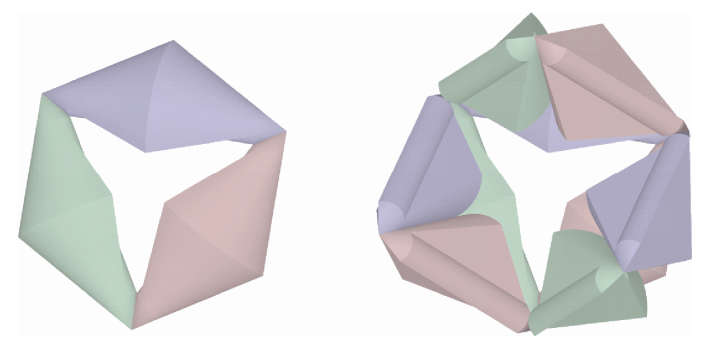

图 14 转子的摆放

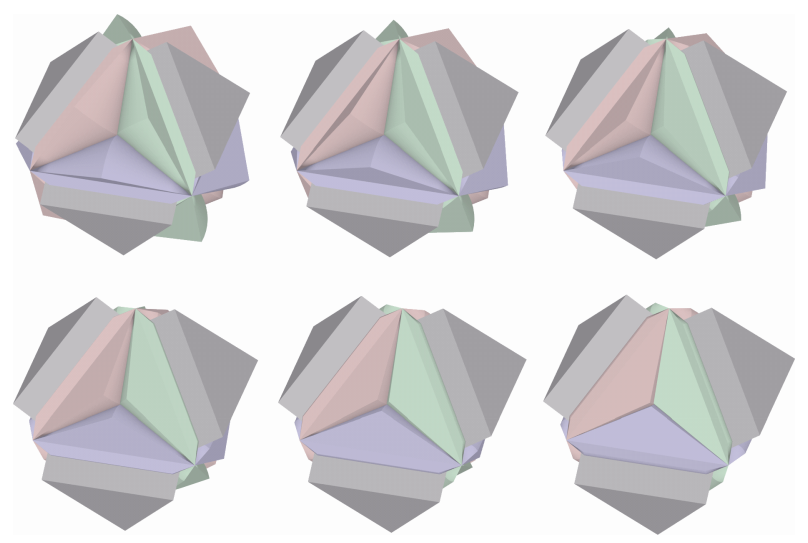

图 15 模拟动画截图

\section{4 结论}

（1）利用锥形转子和三棱柱活塞构造了一种可 变密闭空间, 且满足空间在变化过程中没有滑动摩 
擦。这个设计的巧妙之处在于利用了正八面体的每 个顶点出发的棱数都是偶数, 以及雉形转子之间能 做到在保持密闭的同时做无滑动的滚动性质。这个 设计的理论意义在于用实例证实了三维空间中构造 无滑动摩擦密闭空间的可行性。

（2）构造的这种几何结构只需要在柱形活塞上 增加止回阀和和固定并保持转子和同步运动的连动 机构即可设计机械损耗极小的容积泵, 因而完全可 以根据需求投入使用。

(3) 本文的设计以非常小的磨损产生较高的压 强的性质, 可以用来构造压缩机 ${ }^{[20]}$ 、人造心脏等; 它在能量转化中的机械损耗小的性质, 它可以用来 构造液压或气动马达 ${ }^{[21]}$ 。由此可见, 这种设计在未 来的制造业领域有着非常广阔的应用前景。

\section{参 考 文 献}

[1] CRITCHLEY L A H, JULIAN A J H C. A meta-analysis of studies using bias and precision statistics to compare cardiac output measurement techniques[J]. Journal of Clinical Monitoring and Computing, 1999, 15(2): 85-91.

[2] HARPER L E. Positive displacement pump: U.S. Patent No. 2, 308, 974[P]. 1943-01-19.

[3] LEFFERSON P. Constant pressure pump: U.S. Patent No. 3, 985, 467[P]. 1976-10-12.

[4] JOSÉ G, JOAQUIN F, BLANCO E, et al. Numerical simulation of the dynamic effects due to impeller-volute interaction in a centrifugal pump[J]. Journal of Fluids Engineering, 2002, 124(2): 348-355.

[5] AUGUSTIN P F. Refrigerating machine: U.S. Patent No. 1, 835, 081[P].1931-12-08.

[6] van BORK, ERIK C. Computer controlled metering pump: U.S. Patent No. 5, 056, 036[P]. 1991-10-08.

[7] CAVIL L. Vane pump seal: U.S. Patent Application 16/144, 074[P]. 2019-1-24.

[8] MIKKELSEN S. Pump seal leakage detection system: U.S. Patent Application 16/135, 419[P]. 2019-3-21.

[9] MILLER J E. The reciprocating pump: Theory, design, and use[M]. London: Wiley-Interscience, 1987.

[10] MANRING N D, SURESH B K. The the-oretical flow ripple of an external gear pump[J]. Journal of Dynamic Systems, Measurement, and Control, 2003, 125(3): 396-404.

[11] COOPER P, ALLAN J P. Sealless multiphase screw-pump- and-motor package: U.S. Patent No. 6, 457, 950[P]. 2002-10-01.

[12] PERSSON B N J. Sliding friction: Physical principles and applications $[\mathrm{M}]$. New York: Springer Science \& Business Media, 2013.

[13] TABOR D. The mechanism of rolling friction II. The elastic range[C]// Proc. R. Soc. Lond. A， 1955，229: 198-220.

[14] Blender Foundation. Negative-stiffness-mechanism vibration isolation system[C]// Proceedings of the SPIE-the International Society for Optical Engineering, 1999, 98-105.

[15] DARDE A. Air separation and flue gas com-pression and purification units for oxy-coal combustion systems[J]. Energy Procedia, 2009(1): 527-534.

[16] LUND H, GEORGES S. The role of compressed air energy storage (CAES) in future sustainable energy systems $[\mathrm{C}] / /$ Energy Conversion and Management 50.5, 2009: 1172-1179.

[17] HALAMAY D A. Reserve requirement impacts of large-scale integration of wind, solar, and ocean wave power generation[J]. IEEE Transactions on Sustainable Energy, 2011, 2(3): 321-328.

[18] ZEISCHEGG W. Regular and semi-regular polyhedrons constructed from polyhedral components: U.S. Patent No. 3 , 659, 360[P].1972-05-02.

[19] 张彩明, 杨兴强, 李学庆. 计算机图形学 [M]. 北京: 科学出版社, 2005 .

ZHANG Caiming, YANG Xingqiang, LI Xueqing. Computer graphics [M]. Beijing: Science Press, 2005.

[20] WOOLLENWEBER, W E, EDWARD M H. Mo-tor-driven centrifugal air compressor with internal cooling airflow: U.S. Patent No. 6, 102, 672[P]. 2000-08-15.

[21] PADTURE, N P, MAURICE G, ERIC H J. Thermal barrier coatings for gas-turbine engine applications[J]. Science, 2002, 296(5566): 280-284.

作者简介: 马龙, 男, 1985 年出生, 博士。主要研究方向为几何建模。 E-mail: ma long@ntu.edu.sg; malongsdu1985@hotmail.com 贺英, 男, 1974 年出生, 博士, 副教授, 博士研究生导师。主要研究方 向为可视计算。

E-mail: yhe@ntu.edu.sg

周元峰, 男, 1980 年出生, 博士, 教授, 博士研究生导师。主要研究方 向为智能图形图像处理、几何造型、计算几何及医学影像处理。

E-mail: yfzhou@sdu.edu.cn

张彩明, 男, 1955 年出生, 博士, 教授, 博士研究生导师。主要研究方向 为可视媒体。

E-mail: czhang@sdu.edu.cn 